\title{
Spin-Wave Diode
}

\author{
Jin Lan (兰金), ${ }^{1}$ Weichao Yu (余伟超), ${ }^{1}$ Ruqian Wu, ${ }^{1,2}$ and Jiang Xiao (萧江) ${ }^{1,3, *}$ \\ ${ }^{1}$ Department of Physics and State Key Laboratory of Surface Physics, \\ Fudan University, Shanghai 200433, China \\ ${ }^{2}$ Department of Physics and Astronomy, University of California, Irvine, California 92697-4575, USA \\ ${ }^{3}$ Collaborative Innovation Center of Advanced Microstructures, Fudan University, \\ Shanghai 200433, China
}

(Received 31 July 2015; published 28 December 2015)

\begin{abstract}
A diode, a device allowing unidirectional signal transmission, is a fundamental element of logic structures, and it lies at the heart of modern information systems. The spin wave or magnon, representing a collective quasiparticle excitation of the magnetic order in magnetic materials, is a promising candidate for an information carrier for the next-generation energy-saving technologies. Here, we propose a scalable and reprogrammable pure spin-wave logic hardware architecture using domain walls and surface anisotropy stripes as waveguides on a single magnetic wafer. We demonstrate theoretically the design principle of the simplest logic component, a spin-wave diode, utilizing the chiral bound states in a magnetic domain wall with a Dzyaloshinskii-Moriya interaction, and confirm its performance through micromagnetic simulations. Our findings open a new vista for realizing different types of pure spin-wave logic components and finally achieving an energy-efficient and hardware-reprogrammable spin-wave computer.
\end{abstract}

DOI: 10.1103/PhysRevX.5.041049

\section{INTRODUCTION}

In the post silicon era, Moore's law is not sustainable, partly because of the power consumption caused by the Joule heating from the electric current. To avoid the unmanageable power dissipation, scientists have been trying to use various (quasi)particles other than electrons as information carriers, such as photons in photonics [1], electron spin in spintronics [2], phonons in phononics [3,4], and spin waves in magnonics [5-8]. Among these efforts, magnonics, which can be realized in insulators, is particularly interesting mainly because of its energy-saving benefit since spin waves produce no Joule heating. Since both spin waves and magnetic memory are associated with the reordering of magnetic moments, it is possible and natural to integrate both logic and storage operations through pure spin-wave information processing without the need for other information architectures. New magnonics hardware architecture designs, as we propose below, allow magnonics to be realized on a single magnetic thin film-a magnetic wafer using its "soft" magnetic structures. Such an integrated spin-wave circuit is reprogrammable by repatterning the magnetic texture. This is in contrast to

\footnotetext{
*Corresponding author. xiaojiang@fudan.edu.cn

Published by the American Physical Society under the terms of the Creative Commons Attribution 3.0 License. Further distribution of this work must maintain attribution to the author(s) and the published article's title, journal citation, and DOI.
}

Subject Areas: Condensed Matter Physics,

Magnetism, Materials Science most present-day electronic technologies that use the "hard" physical structures consisting of several materials and layers.

Controlling the information transmission direction is a basic feature of all information processing systems, as implemented in various diode structures. In addition to the classical electric diode using the p-n junction, there are optical diodes [9], heat diodes [10,11], acoustic-wave (phonon) diodes [12,13], and spin-Seebeck diodes [14], etc. In this paper, we propose a design of the spin-wave diode utilizing the spatial separation of the spin-wave bound states caused by the Dzyaloshinskii-Moriya interaction (DMI) $[15,16]$ within a magnetic domain wall. The DMI is an antisymmetric exchange coupling induced by spin-orbit interaction in magnetic materials with broken inversion symmetry, either in a bulk lattice or at the interface. The functionality of this reprogrammable spinwave diode is confirmed by micromagnetic simulations.

\section{MAGNETIC-WAFER-BASED SPIN-WAVE ARCHITECTURE}

To construct spin-wave logic components on a twodimensional magnetic wafer, we first need waveguides or wires that can transport spin waves. A magnetic domain wall can be a natural waveguide using the domain-wall bound state as a carrier, as demonstrated numerically by Garcia-Sanchez et al. [17]. Another type of waveguide utilizes the surface spin-wave mode induced by the easy-axis surface anisotropy (EASA) [18,19], with which 


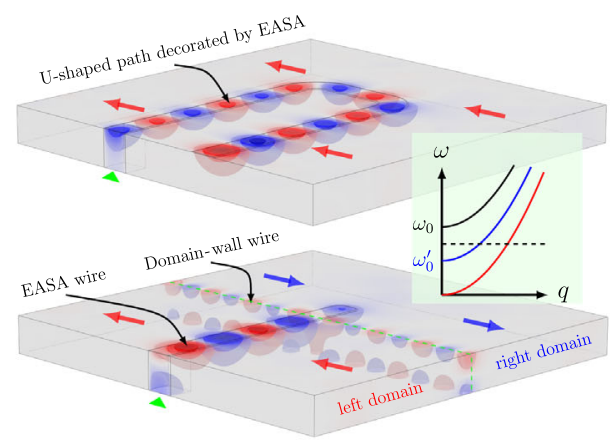

(a) EASA wire and its crossing with a domain-wall wire

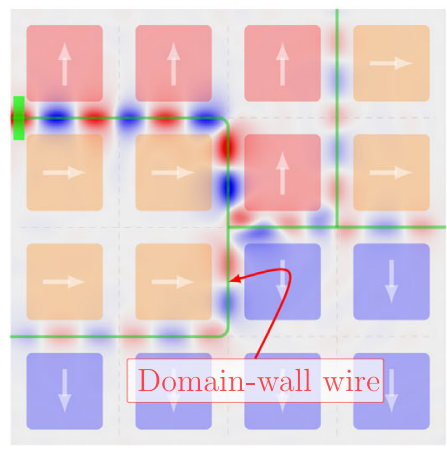

(b) Domain-wall circuit

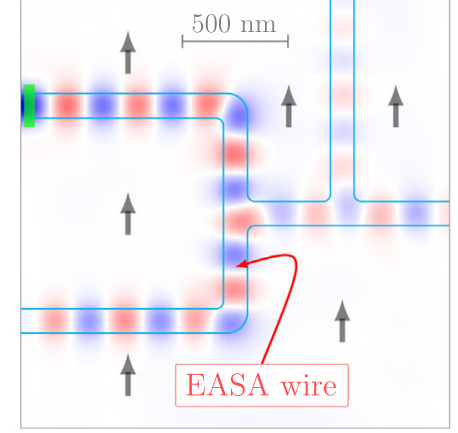

(c) EASA circuit

FIG. 1. The domain-wall circuit and EASA circuit. (a) Upper diagram: simulated EASA-induced surface spin-wave mode propagating along a U-shaped path decorated by EASA. Lower diagram: Simulated spin-wave interconnection from an EASA wire to a domain-wall wire. Both images are simulated on a homogeneous magnetic wafer of size $1600 \mathrm{~nm} \times 1600 \mathrm{~nm} \times 150 \mathrm{~nm}$ with spin-wave excitation at the position indicated by the triangle. Inset: The dispersion relation for the bulk spin wave (black), EASA surface mode (blue), and domain-wall bound state (red); the excitation frequency is indicated by the dashed line lying below the bulk gap and above the surface-wave gap. (b) Simulated spin-wave propagation in a domain-wall circuit made of the domain-wall wires printed on a magnetic wafer with a biaxial anisotropy and four possible domain orientations, and wafer size $2000 \mathrm{~nm} \times 2000 \mathrm{~nm} \times 10 \mathrm{~nm}$. (c) Simulated EASA-induced surface spin wave transporting through an EASA circuit in a homogeneous magnetic wafer of size $2000 \mathrm{~nm} \times 2000 \mathrm{~nm} \times 30 \mathrm{~nm}$.

the surface spins tend to point in the surface normal direction [20]. To construct an EASA wire, the surface of the magnetic wafer is decorated by EASA along the wire path for the surface mode to propagate. Such a decoration can be either a capping layer of other materials or simply a process that modifies the original surface structure. The penetration depth of this EASA surface mode is inversely proportional to the strength of EASA; i.e., the stronger the EASA, the shallower the penetration [18,19]. Neither type of spin-wave waveguide has a hard structure on the magnetic wafer, especially the domain-wall wire, which can even be moved to another position. The domain-wall wire and EASA wire can work simply because the dispersions for the domain-wall bound state and EASA surface state have either no gap or a smaller gap than the bulk spin waves. Within domains, ignoring the dipolar coupling, the bulk spin-wave dispersion is $\omega_{\text {Bulk }}=$ $\omega_{0}+A_{\mathrm{ex}} q^{2}$ [the top black curve in the inset of Fig. 1(a)], where $\omega_{0}$ is the spin-wave gap in the bulk, $A_{\mathrm{ex}}$ is the exchange coupling constant, and $q$ is the in-plane wave vector. The domain-wall bound state is gapless, and its dispersion is $\omega_{\mathrm{BS}}=A_{\mathrm{ex}} q^{2}$ [the bottom red curve in the inset of Fig. 1(a)] [21,22]. The dispersion of the EASA surface spin wave is approximately $\omega_{\text {EASA }}=\omega_{0}^{\prime}+A_{\text {ex }} q^{2}$ with $\omega_{0}^{\prime}<\omega_{0}$ [middle blue curve in the inset of Fig. 1(a)] [18]. The spin-wave modes whose frequency $\omega$ lies within the range $\omega_{0}^{\prime}<\omega<\omega_{0}$ can only propagate in the domainwall wire and the EASA wire, but not in the bulk. Through micromagnetic simulations, we demonstrate in Fig. 1(a) the transport of spin waves in a U-shaped EASA wire (upper) and across an interconnection (lower) between an EASA wire and a domain-wall wire.
Making use of the spin-wave wires, an integrated spinwave circuit can be imprinted onto a magnetic wafer. As an example, for a magnetic wafer with biaxial anisotropy along two perpendicular axes, we may create an artificial chessboardlike pattern such that in each square the magnetic order can point in any of the four possible orientations, as shown in Fig. 1(b). The domain walls are pinned along the grid lines, which can be carved notches on the wafer surface. Such chessboardlike magnetic structure can be used as twodimensional memory similar to the one-dimensional racetrack memory [23]. However, instead of using magnetic domains to store information, we propose to use domain walls as spin-wave wires, which can be interconnected to form a domain-wall circuit. Not only can the domain-wall circuit be imprinted in any pattern, but it can also be rewritten by reorienting the magnetization direction of each square. Therefore, this type of chessboard domain-wall circuit is extremely flexible to construct a large number of different spin-wave circuits. Simpler circuits can be realized in a wafer with uniaxial anisotropy. It is also possible to use EASA wires to build a spin-wave circuit: On a magnetic wafer, EASA wires can be imprinted into any pattern of circuits by simply decorating the surface of the circuits by EASA, as shown in Fig. 1(c). A more general design may use a hybrid circuit that contains both domain-wall and EASA wires.

\section{SPIN-WAVE DIODE}

To demonstrate the concept of our design of a spin-wave diode, we use the magnetic wafer that has uniaxial anisotropy along the $y$ direction and that contains two magnetic domains with a Bloch domain wall in between, as shown in Fig. 2(a), where the magnetization in the left or 


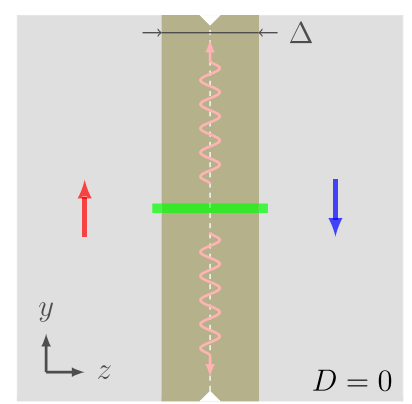

(a) Domain wall without DMI

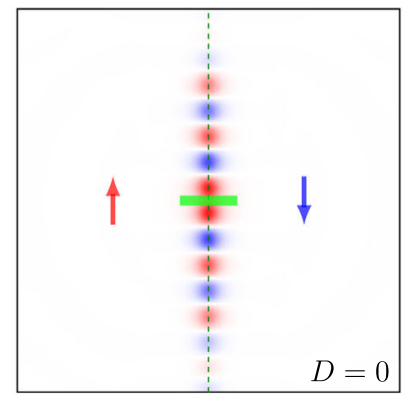

(e) Domain wall without DMI

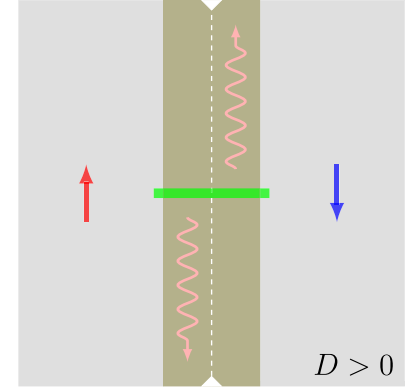

(b) Domain wall with DMI

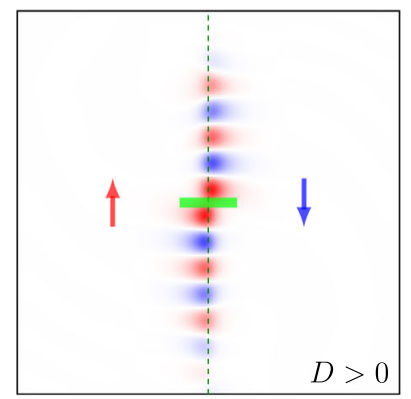

(f) Domain wall with DMI

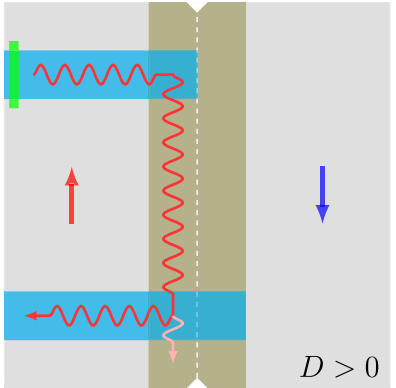

(c) Diode - forward direction

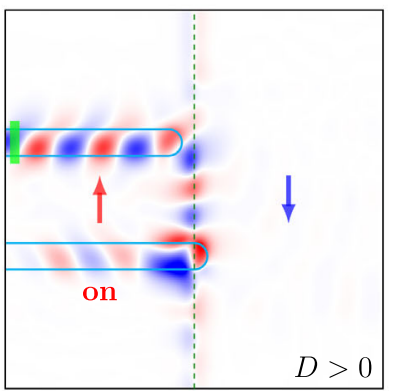

(g) Diode - forward direction

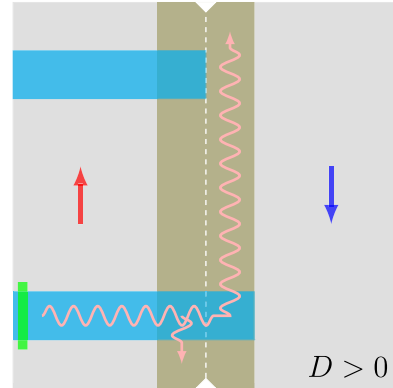

(d) Diode - reverse direction



(h) Diode - reverse direction

FIG. 2. The design of the diode and the simulations. Top row: The design of the spin-wave diode. (a) Domain-wall wire without DMI. The bound spin-wave state propagates in both directions identically, where the domain-wall region is shaded with darker brown color and is pinned at the kink position. (b) Domain-wall wire with DMI. The bound states propagating upward or downward are spatially shifted to the right or left side of the wall. (c) Spin-wave diode-forward direction. The spin wave transmits from the upper to the lower terminal. (d) Spin-wave diode-reverse direction. The spin wave is blocked from the lower to the upper terminal. The wavy lines denote the route of the spin-wave propagation. Bottom row: Numerical micromagnetic simulations of the spin-wave diode (color map of $m_{z}$ ). (e) Without DMI, the bound spin-wave state travels symmetrically. (f) With DMI, the bound spin-wave states become chiral. (g) Spin-wave diode - the forward direction. (h) Spin-wave diode - the reverse direction. In all panels, the little green bar indicates the spin-wave injection location where an oscillating magnetic field is applied.

right domain points in the $\pm y$ direction, respectively. The magnetization within the wall evolves from $y$ to $-y$ by rotating out of plane in the $x$ direction. When there is no DMI, the bound spin-wave state propagates identically to both the $\pm y$ directions along the domain-wall wire [see Fig. 2(a)]. However, the presence of DMI effectively applies a magnetic field in the domain-wall region, as shown below, and the bound spin-wave states that propagate in opposite directions are spatially separated towards two edges of the wire, as illustrated in Fig. 2(b).

\section{A. Design principle}

This spatial separation feature allows us to design the spin-wave diode, as shown in Figs. 2(c) and 2(d). The two terminals patterned on the left side of the domain wall (indicated by light blue rectangles) are made of EASA wires. The upper terminal only overlaps with the left half of the domain wall, while the lower terminal covers the whole domain-wall width. The diode works as follows: In the forward direction, the spin wave is injected from the upper terminal, as shown in Fig. 2(c). The two terminals are connected via the left half of the domain-wall wire because the down-going spin wave is localized on the left half of the wall. However, in the reverse direction when the spin wave is injected from the lower terminal, the up-going spin wave is localized on the right half of the domain wall and hence cannot reach the upper terminal, as shown in Fig. 2(d). This unidirectional transport of spin waves is clearly the diode effect.

The operation of the spin-wave diode is confirmed by numerical micromagnetic simulations using an yttrium iron garnet (YIG) thin film of thickness $30 \mathrm{~nm}$, with parameters given in Sec. V. Figures 2(e) and 2(f) show the spatial shift of the spin-wave bound states traveling in $+y$ and $-y$ direction in the domain-wall wire under the influence of DMI. Figures 2(g) and 2(h) show the diode effect for the forward and reverse spin-wave propagations, a behavior that confirms our analyses above. The power loss in the forward direction is about $9.5 \mathrm{~dB}$ (power decreased by 9 times), while the power loss in the reverse direction is about $24 \mathrm{~dB}$ (power decreased by 257 times), and the on/off power-loss difference is $14.5 \mathrm{~dB}$; therefore, the power loss in the reverse direction is much larger than in the forward direction. In these simulations, we artificially set high damping above and below the diode terminals to eliminate the boundary effects. 
The experimental realization of the spin-wave diode structure proposed in Figs. 2(c) and 2(d) can follow a hardfirst-magnetic-second procedure; i.e., the terminals and the notches that pin the domain wall can be patterned first, and the positioning of such hard structures is easy to control with present nanofabrication technology. The magnetic domain-wall structure is prepared afterwards such that the domain wall will move to the desired position at the notches by itself. This procedure saves us the trouble of finding the domain wall.

All simulation figures shown in the paper ignore the dipolar fields. However, we tested the influence of the dipolar fields and confirmed that the spatial separation of the bound spin-wave states and the consequent spin-wave diode effect are robust against such fields (see Ref. [24]).

\section{B. Theory}

Obviously, the crucial ingredient for realizing the spinwave diode effect is the spatial separation of the spin-wave bound states caused by the DMI in the domain wall. To prove this, we adopt the bulk form of DM energy $E_{\mathrm{DMI}}=D \mathbf{m} \cdot(\nabla \times \mathbf{m})$, for which the Bloch domain wall is favored against the Néel wall. We can understand the chiral feature of the bound states in a semiclassical way by transforming the equation of motion for spin-wave dynamics into an effective Schrödinger equation [22]. The spinwave dynamics is governed by the Landau-Lifshitz-Gilbert (LLG) equation:

$$
\dot{\mathbf{m}}=-\gamma \mathbf{m} \times \mathbf{H}_{\text {eff }}+\alpha \mathbf{m} \times \dot{\mathbf{m}},
$$

where $\mathbf{m}(\mathbf{r}, t)$ is the unit vector in the direction of the magnetization, $\alpha$ is the Gilbert damping parameter, and $\gamma \mathbf{H}_{\text {eff }}=K m_{y} \hat{\mathbf{y}}+A_{\text {ex }} \nabla^{2} \mathbf{m}-D \nabla \times \mathbf{m}$ is the effective magnetic field acting on $\mathbf{m}$ because of contributions from anisotropy (along $\hat{\mathbf{y}}$ ), exchange, and DMI. In the absence of DMI, the domain-wall width is determined as $\Delta=\sqrt{A_{\mathrm{ex}} / K}$, which is the same for the Bloch and Néel domain walls. When DMI is included, the domain wall twists slightly for the Néel wall [25] but remains unchanged for the Bloch wall. Let $\hat{\mathbf{m}}_{0}=$ $\left(\sin \theta_{0} \cos \phi_{0}, \sin \theta_{0} \sin \phi_{0}, \cos \theta_{0}\right)$ be the static magnetic texture of the domain wall along $\hat{\mathbf{z}}$, where $\theta_{0}(z)$ and $\phi_{0}(z)$ are the polar and azimuthal angles of $\hat{\mathbf{m}}_{0}(z)$ with respect to the $\hat{\mathbf{z}}$ axis. Let $\delta \mathbf{m}=m_{\theta} \hat{\mathbf{e}}_{\theta}+m_{\phi} \hat{\mathbf{e}}_{\phi}$ be the spin-wave excitation on top of the static $\hat{\mathbf{m}}_{0}$, where $\hat{\mathbf{e}}_{\theta}, \hat{\mathbf{e}}_{\phi} \perp \hat{\mathbf{m}}_{0}$ are the two transverse directions to $\hat{\mathbf{m}}_{0}$. As for the dynamics of $\delta \mathbf{m}$, previous studies show that the effect of the inhomogeneous magnetization texture on $\delta \mathbf{m}$ can be represented by a scalar potential [22], while the effect of DMI can be represented by a vector potential [26].

The static structure of a Bloch domain wall is unmodified by the DMI and still takes the Walker profile: $\theta_{0}(z)=\pi / 2, \quad \phi_{0}(z)=\pi / 2+2 \arctan [\exp (z / \Delta)]$, where the magnetization within the wall rotates in the $x$-y plane



FIG. 3. The spatial profile for the effective scalar potential and the effective magnetic field. We show the spatial distribution of the scalar potential $V(z)$ and the magnetic field $B(z)$ in the $y-z$ plane. The density map is the scalar potential, and the disk size represents the magnitude of the magnetic field pointing in the $x$ direction.

in a certain direction depending on the sign of $D$ [27]. By redefining $\psi=m_{\theta}-i m_{\phi}$, the LLG equation (1) governing the dynamics of $\delta \mathbf{m}$ can be recast into an effective Schrödinger equation for $\psi$ :

$$
i \hbar \frac{\partial}{\partial t} \psi=\left[\frac{1}{2 m^{*}}\left(\hat{\mathbf{p}}-\frac{q}{c} \mathbf{A}\right)^{2}+V\right] \psi
$$

where the effective mass $m^{*}=\hbar / 2 A_{\mathrm{ex}}$, the momentum operator $\hat{\mathbf{p}}=-i \hbar \nabla$, the scalar potential $V=-\hbar K \cos 2 \phi_{0}$, and the vector potential $\mathbf{A}=-\left(D m^{*} c / q\right) \sin \phi_{0} \hat{\mathbf{z}}$, which corresponds to an effective magnetic field perpendicular to the $y-z$ plane: $\mathbf{B}=\nabla \times \mathbf{A}=\left(D m^{*} c / q\right) \phi_{0}^{\prime} \cos \phi_{0} \hat{\mathbf{x}}$. Therefore, the behavior of the spin wave in a Bloch domain-wall structure is equivalent to the motion of a charged particle with mass $m^{*}$ and charge $q$ in a potential well $V(z)$ and magnetic field $\mathbf{B}(z) \| \hat{\mathbf{x}}$. The spatial profiles for the potential $V$ and magnetic field $\mathbf{B}$ are plotted in Fig. 3. The potential well $V(z)$ is special, not only because of its reflectionlessness but also for the existence of a bound state at the bottom of the potential well with zero energy in the $z$ direction.

Based on the effective Schrödinger equation (2), we may understand the transport behavior of the bound states within domain walls in a semiclassical way: (a) For $D=0$, the bound state only feels the potential well $V(z)$ with a vanishing magnetic field $\mathbf{B}=0$, so it is confined in the $z$ direction within the well and travels along the domain wall in the $\pm y$ directions symmetrically, as shown in Fig. 2(a); (b) however, for $D>0$, the effective magnetic field $\mathbf{B}$ pointing in the $\hat{\mathbf{x}}$ direction (perpendicular to the thin film) is nonzero and is maximized at the domainwall center (see Fig. 3). Consequently, the spin wave 


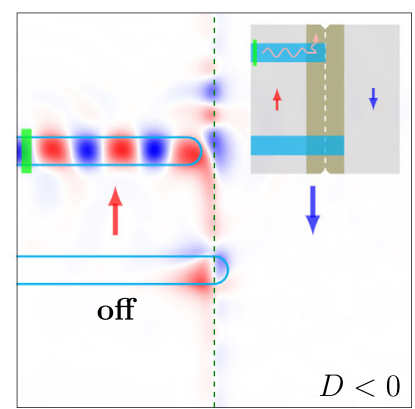

(a) Reverse direction



(b) Forward direction
FIG. 4. Reprogramming the diode by changing the sign of the DMI parameter. The forward direction of the spin-wave diode for $D<0$ is opposite to that for $D>0$ in Figs. 2(g) and 2(h). The insets on the top right are the spin-wave propagation diagrams.

moving upwards to $+y$ (downwards to $-y$ ) bends to the right (left) because of the effective Lorentz force [see Fig. 2(b)]. A head-to-head Néel domain wall can also be stabilized by the DMI, but it does not have the spatial separation behavior because the corresponding vector potential $\mathbf{A}$ is proportional to $D^{2}$, much weaker than a Bloch wall.

\section{Reprogrammability}

If DMI originates from the inversion symmetry breaking at the surface in magnetic thin films, the spin-wave diode works just as well, but for a head-to-head Néel domain wall instead. The advantage of interfacial DMI is its tunability by an external electric field, which can tune the magnitude and even the sign of the DMI parameter $D$. The electricfield-controlled DMI has been theoretically proposed by Liu et al. [28] and experimentally confirmed by Zhang et al. [29], both in YIG. In our system, the $D$ can be tuned by applying a gating voltage throughout the whole thin film. When $D$ changes sign $(D<0)$, the direction of the effective magnetic field $\mathbf{B}$ also changes from $\hat{\mathbf{x}}$ to $-\hat{\mathbf{x}}$, so the spin wave moving to $+y(-y)$ now bends to the left (right), opposite to that in Figs. 2(b) and 2(f). Consequently, the forward direction of the spin-wave diode also changes, as shown in Fig. 4. In fact, by continuously tuning the value and sign of $D$ using a perpendicular electric field, the spinwave diode can have (i) $\mathrm{L} \rightarrow \mathrm{R}$ transmission only $(D>0)$, (ii) $\mathrm{R} \rightarrow \mathrm{L}$ transmission only $(D<0)$, or (iii) $\mathrm{L} \leftrightarrow \mathrm{R}$ transmission $(D \simeq 0)$. We demonstrate the real-time switching of the three stages of transmission by continuously tuning parameter $D$ in a movie in Ref. [24].

Another way of tuning the functionality of the spin-wave diode is to shift the domain-wall position, which can be realized in various ways, for instance, by applying a magnetic field, or via current-induced spin-transfer torque, or purely by a spin wave with magnonic spin-transfer torque. For this purpose, we design a two-sided spin-wave diode, as shown in Fig. 5, where the terminals are connected to the domain wall from the opposite sides instead of the same side as in Fig. 2. Figure 5 shows the functionality of the device for five different domain-wall

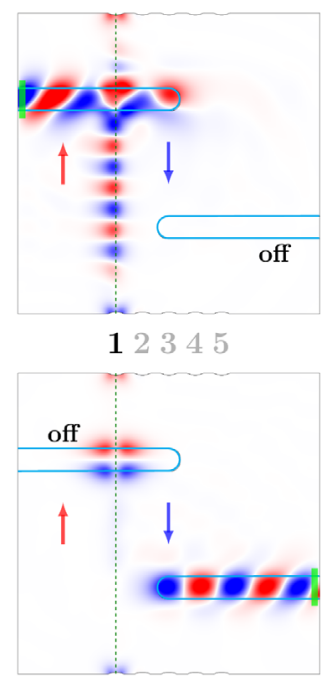

(a)

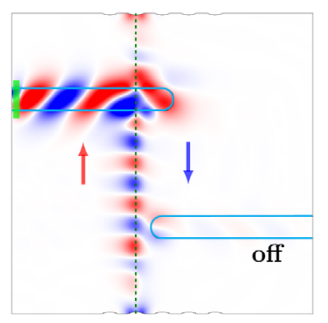

12345

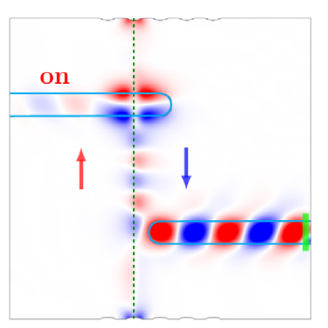

(b)

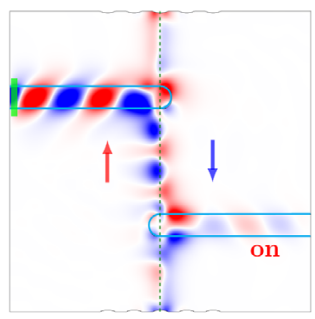

$12 \mathbf{3} 45$

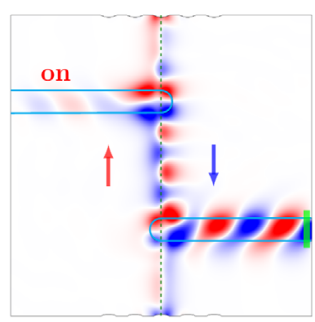

(c)

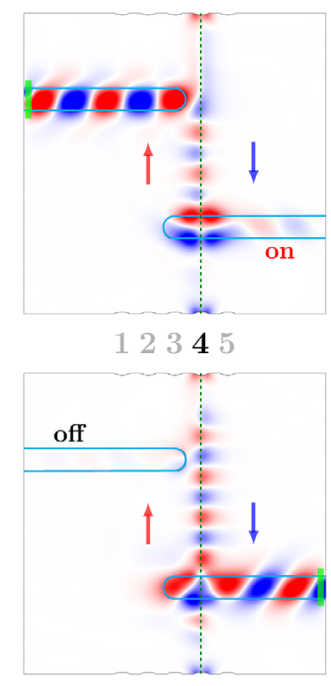

(d)

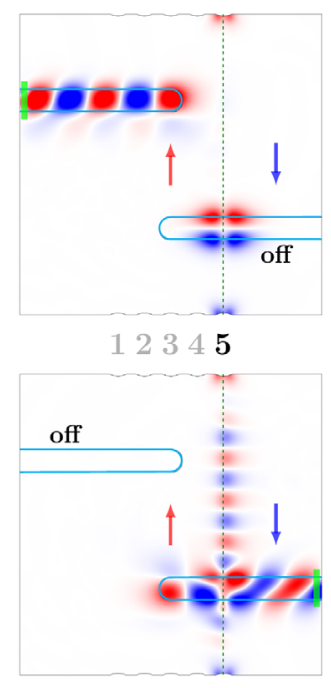

(e)

FIG. 5. Reprogramming the diode by moving the domain-wall position. The functionality is tuned by the domain-wall position (from position 1 to 5, the domain-wall center is indicated by the dashed line). The spin wave is injected from the left (right) terminal for the upper (lower) row. (a) Position 1, off in both directions. (b) Position 2, diode with the forward direction from right to left. (c) Position 3 , on in both directions. (d) Position 4, diode with the forward direction from left to right, opposite to position 2. (e) Position 5, off in both directions, similar to position 1 . The kinks are to pin the domain walls. The simulation is performed on a two-dimensional film of size $1600 \mathrm{~nm} \times 1600 \mathrm{~nm}$ with an additional perpendicular anisotropy in the terminal area to mimic the EASA in three-dimensional samples. (See the movie in Ref. [24] for simulated real-time switching among these functions by current-induced domain-wall motion.) 
positions pinned by the five kinks. Depending on the domain-wall position, the two terminals can either be completely disconnected, manifest the diode effect, or be connected in both ways. Therefore, by repositioning the domain wall, the device function can be easily modified. In Ref. [24], we demonstrate the real-time change of the spinwave transmission properties due to the domain-wall motion caused by a spin current.

\section{CONCLUSIONS}

In conclusion, we proposed a reprogrammable magnonic hardware architecture on a single magnetic wafer based on two types of waveguides using domain walls and EASA stripes. Utilizing the chiral property from DMI in the domain-wall wire, we demonstrated by micromagnetic simulations the first building block on this spin-wave architecture-a spin-wave diode. Our findings open the door for reprogrammable pure spin-wave circuits on a single magnetic wafer and for ultimately realizing a magnonic computer.

\section{METHODS}

The simulation is performed in COMSOL Multiphysics using the mathematical module where the LLG equation is transformed into weak form and solved by the generalized alpha method (amplification of high frequency is 0.6) in a three-dimensional environment. The sample is an YIG thin film (size $1600 \mathrm{~nm} \times 1600 \mathrm{~nm} \times 30 \mathrm{~nm}$ if not mentioned otherwise). The parameters for YIG are as follows: the anisotropy of the magnetic wafer $K / \gamma=3.88 \times 10^{4} \mathrm{~A} / \mathrm{m}$, exchange coefficient $A_{\mathrm{ex}} / \gamma=3.28 \times 10^{-11} \mathrm{~A} \cdot \mathrm{m}$, the gyromagnetic ratio $\gamma=2.21 \times 10^{5} \mathrm{~Hz} /(\mathrm{A} / \mathrm{m})$ [22], and the DMI coefficient $D / \gamma=1.0 \times 10^{-3}$ A. The thickness of the EASA layer is $3 \mathrm{~nm}$ and $K_{s} / \gamma=1.0 \times 10^{-3} \mathrm{~A}$. In order to stabilize the domain wall, a hard-axis anisotropy in the $z$ direction, $K_{h} / \gamma=1.0 \times 10^{5} \mathrm{~A} / \mathrm{m}$, is applied, and a groove with a thickness of $10 \mathrm{~nm}$ is made to pin the domain wall. The frequency of the oscillating field $f=2 \mathrm{GHz}$ is applied locally at the position indicated by the green bar in each simulation figure. The damping coefficient in the working area $\alpha=10^{-4}$, while the damping near the boundary is set to $\alpha=0.5$ to eliminate boundary effects such as reflections. In the simulation of Fig. 5, the additional perpendicular anisotropy in the terminal area is taken as $K_{s}=0.8 \mathrm{~K}$.

\section{ACKNOWLEDGMENTS}

J. X. thanks Yizheng Wu, Donglai Feng, and Lei Zhou for helpful remarks on the manuscript. This work was supported by the National Natural Science Foundation of China (Grants No. 11474065 and No. 91121002), the National Basic Research Program of China (Grants No. 2014CB921600, No. 2011CB925601, and No. 2015CB921400). R. W. also acknowledges support from the 1000-talent program. Work at
UCI was supported as part of SHINES, an Energy Frontier Research Center funded by the U.S. Department of Energy, Office of Science, Basic Energy Sciences under Grant No. SC0012670.

J. L. and W. Y. contributed equally to this work.

[1] J. L. O'Brien, A. Furusawa, and J. Vučković, Photonic Quantum Technologies, Nat. Photonics 3, 687 (2009).

[2] F. Pulizzi, Spintronics, Nat. Mater. 11, 367 (2012).

[3] N. Li, J. Ren, L. Wang, G. Zhang, P. Hänggi, and B. Li, Phononics: Manipulating Heat Flow with Electronic Analogs and Beyond, Rev. Mod. Phys. 84, 1045 (2012).

[4] M. Maldovan, Sound and Heat Revolutions in Phononics, Nature (London) 503, 209 (2013).

[5] A. A. Serga, A. V. Chumak, and B. Hillebrands, YIG Magnonics, J. Phys. D 43, 264002 (2010).

[6] V. V. Kruglyak, S. O. Demokritov, and D. Grundler, Magnonics, J. Phys. D 43, 264001 (2010).

[7] A. Khitun, M. Bao, and K. L. Wang, Magnonic Logic Circuits, J. Phys. D 43, 264005 (2010).

[8] B. Lenk, H. Ulrichs, F. Garbs, and M. Münzenberg, The Building Blocks of Magnonics, Phys. Rep. 507, 107 (2011).

[9] M. D. Tocci, M. J. Bloemer, M. Scalora, J. P. Dowling, and C. M. Bowden, Thin-Film Nonlinear Optical Diode, Appl. Phys. Lett. 66, 2324 (1995).

[10] B. Li, L. Wang, and G. Casati, Thermal Diode: Rectification of Heat Flux, Phys. Rev. Lett. 93, 184301 (2004).

[11] C. W. Chang, D. Okawa, A. Majumdar, and A. Zettl, Solid-State Thermal Rectifier, Science 314, 1121 (2006).

[12] B. Liang, B. Yuan, and J.-c. Cheng, Acoustic Diode: Rectification of Acoustic Energy Flux in One-Dimensional Systems, Phys. Rev. Lett. 103, 104301 (2009).

[13] B. Liang, X. S. Guo, J. Tu, D. Zhang, and J. C. Cheng, An Acoustic Rectifier, Nat. Mater. 9, 989 (2010).

[14] S. Borlenghi, W. Wang, H. Fangohr, L. Bergqvist, and A. Delin, Designing a Spin-Seebeck Diode, Phys. Rev. Lett. 112, 047203 (2014).

[15] I. Dzyaloshinsky, A Thermodynamic Theory of, Weak, Ferromagnetism of Antiferromagnetics, J. Phys. Chem. Solids 4, 241 (1958).

[16] T. Moriya, Anisotropic Superexchange Interaction and Weak Ferromagnetism, Phys. Rev. 120, 91 (1960).

[17] F. Garcia-Sanchez, P. Borys, R. Soucaille, J.-P. Adam, R. L. Stamps, and J.-V. Kim, Narrow Magnonic Waveguides Based on Domain Walls, Phys. Rev. Lett. 114, 247206 (2015).

[18] J. Xiao and G. E. W. Bauer, Spin-Wave Excitation in Magnetic Insulators by Spin-Transfer Torque, Phys. Rev. Lett. 108, 217204 (2012).

[19] Y. Zhou, H. Jiao, Y.-t. Chen, G. E. W. Bauer, and J. Xiao, Current-Induced Spin-Wave Excitation in Pt/YIG Bilayer, Phys. Rev. B 88, 184403 (2013).

[20] A. G. Gurevich and G. A. Melkov, Magnetization Oscillations and Waves (CRC Press, Boca Raton, 1996).

[21] R. K. Dodd, Solitons and Nonlinear Wave Equations (Academic Press, 1982). 
[22] P. Yan, X.S. Wang, and X. R. Wang, All-Magnonic Spin-Transfer Torque and Domain Wall Propagation, Phys. Rev. Lett. 107, 177207 (2011).

[23] S. S. P. Parkin, M. Hayashi, and L. Thomas, Magnetic Domain-Wall Racetrack Memory, Science 320, 190 (2008).

[24] See Supplemental Material at http://link.aps.org/ supplemental/10.1103/PhysRevX.5.041049 for the magnetic numerical simulations with dipolar fields included, and the movies on the real-time change of the spin wave transmissions by tuning the DMI coefficient $D$ or moving the domain wall.

[25] W. Wang, M. Albert, M. Beg, M.-A. Bisotti, D. Chernyshenko, D. Cortés-Ortuño, I. Hawke, and H. Fangohr, Magnon-Driven Domain-Wall Motion with the
Dzyaloshinskii-Moriya Interaction, Phys. Rev. Lett. 114, 087203 (2015).

[26] Y. Onose, T. Ideue, H. Katsura, Y. Shiomi, N. Nagaosa, and Y. Tokura, Observation of the Magnon Hall Effect, Science 329, 297 (2010).

[27] G. Chen, J. Zhu, A. Quesada, J. Li, A. T. N'Diaye, Y. Huo, T. P. Ma, Y. Chen, H. Y. Kwon, C. Won, Z. Q. Qiu, A. K. Schmid, and Y. Z. Wu, Novel Chiral Magnetic Domain Wall Structure in $\mathrm{Fe} / \mathrm{Ni} / \mathrm{Cu}(001)$ Films, Phys. Rev. Lett. 110, 177204 (2013).

[28] T. Liu and G. Vignale, Electric Control of Spin Currents and Spin-Wave Logic, Phys. Rev. Lett. 106, 247203 (2011).

[29] X. Zhang, T. Liu, M. E. Flatté, and H. X. Tang, ElectricField Coupling to Spin Waves in a Centrosymmetric Ferrite, Phys. Rev. Lett. 113, 037202 (2014). 